Research Paper

\title{
Prognostic Value of the Cycle Number of Perioperative Chemotherapy in Locoregionally Advanced Rectal Cancer: a Propensity Score Matching Analysis
}

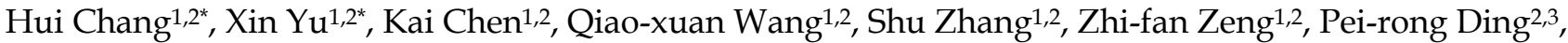 \\ Zhi-zhong Pan ${ }^{2,3}$, Wei-wei Xiao ${ }^{1,2}{ }^{\bowtie}$, Yuan-hong Gao ${ }^{1,2}$ \\ 1. Department of Radiation Oncology, Sun Yat-sen University Cancer Center. \\ 2. State Key Laboratory of Oncology in South China; Collaborative Innovation Center of Cancer Medicine. \\ 3. Department of Colorectal Surgery, Sun Yat-sen University Cancer Center. \\ *Hui Chang and Xin Yu contributed equally to this work. \\ $\square$ Corresponding authors: Prof. Wei-wei Xiao and Prof. Yuan-hong Gao. Department of Radiation Oncology, Sun Yat-sen University Cancer Center. 651 \\ Dongfeng Road East, Guangzhou, Guangdong 510060, China. Prof. Wei-wei Xiao: Tel: +86-13710390520, E-mail: xiaoww@sysucc.org.cn. Prof. Yuan-hong Gao: \\ Tel: +86-13560182168, Fax: +86-020-87343491, E-mail: gaoyh@sysucc.org.cn. \\ ( ) Ivyspring International Publisher. This is an open access article distributed under the terms of the Creative Commons Attribution (CC BY-NC) license \\ (https://creativecommons.org/licenses/by-nc/4.0/). See http://ivyspring.com/terms for full terms and conditions.
}

Received: 2018.05.14; Accepted: 2018.09.05; Published: 2018.10.21

\begin{abstract}
Background: Appropriate cycle number of perioperative chemotherapy for patients with locoregionally advanced rectal cancer (LARC) remains unknown. This study aimed to evaluate how cycle number of perioperative chemotherapy influenced the prognosis of LARC patients.

Methodology I Principal Findings: In this study, a total of 388 consecutive patients were enrolled and retrospectively reviewed if they were diagnosed with untreated stage cll-III LARC and treated with neoadjuvant chemoradiotherapy plus radical surgery followed by adjuvant chemotherapy or not. After grouping by the postoperative pathologic stage (yp0-I vs. ypll-III), propensity score matching was performed in each group to balance baseline characteristics between the patients treated with chemotherapy cycle $\leq 7$ and those treated with chemotherapy cycle $\geq 8$. The chemotherapy cycle was analyzed for its association with the survivals of the matched patients in the 2 groups, respectively. And the incidence of treatment-related complications was also compared. Through analysis, chemotherapy cycle $\geq 8$ appeared to predict better overall, disease-free and distant-metastasis-free survivals in the whole cohort of matched patients ( $P$ values were $0.003,0.002$ and 0.004 , respectively) and the ypll-III group ( $P$ values were $0.006,0.005$ and 0.014 , respectively). But in the yp0-I group, chemotherapy of 8 cycles or more brought no improvement of survivals but only more acute toxicities $(83.5 \%$ vs. $57.0 \%, P<0.001)$.

Conclusions / Significance: Chemotherapy cycle $\geq 8$ was proven associated with improved prognosis of LARC patients, especially those with ypll-III disease. But prolonged chemotherapy should be performed with caution in patients with yp0-I stage.
\end{abstract}

Key words: rectal neoplasms, cycle number, perioperative chemotherapy

\section{Introduction}

Colorectal cancer is one of the main malignancies threatening the health of the Chinese [1]. The most common site of colorectal cancer is rectum, which accounts for nearly $57.2 \%$ of the cases. And $75.6 \%$ of the patients with rectal cancer presented as locoregionally advanced (stage II-III) disease at initial diagnosis [2]. Because of the uneliminable effects in improving resectability, long-term outcome and life quality [3-5], neoadjuvant chemoradiotherapy (NACRT) plus surgery followed by adjuvant chemotherapy (ACT) or not is recommended by the National Comprehensive Cancer Network (NCCN) as 
the standard mode to manage locoregionally advanced rectal cancer (LARC) [6]. Although an ideal local control has now been achieved $[5,7]$, the 5-year overall survival (OS) of LARC remains as 73.3-77.2\% $[5,8]$. Furthermore, it is reported that $20.2-23.9 \%$ of the LARC patients will finally develop distant metastasis $(\mathrm{DM})$ in 3 years $[8,9]$. In other words, the major cause of treatment failure in the era of NACRT is DM, rather than local recurrence (LR).

Systemic therapy, especially chemotherapy, is now the main modality to reduce DM and improve the survival of LARC patients. It is convinced that chemotherapy of enough intensity is needed to eliminate circulating tumor cells, which shed from primary tumor or metastatic lymph nodes to become the seeds of DM lesions [10]. The NCCN guidelines recommend that the standard CAPEOX chemotherapy should be given to a total of 6 months (8 cycles) perioperatively [6]. On the other side, high-intensity chemotherapy might bring severe toxicities which affect patients' life quality, and even cause treatment-related death. Schmoll et al reported that nearly $55 \%$ of the patients developed grade $3 / 4$ toxicities after 8 cycles of CAPEOX chemotherapy [11]. It has been proven that the concentration of circulating tumor cells in a patient depends on his or her tumor burden [12]. So we hypothesized that those with great residual lesions after NACRT might be the suitable population for intensified chemotherapy. Those with little or no residual lesions might not benefit from chemotherapy of such intensity. This study aimed to explore the correlation between the cycle number of perioperative chemotherapy and the prognosis of LARC in patients with different postoperative pathologic stages.

\section{Materials and Methods}

\section{Ethical statement}

This study was approved by the Institutional Review Board of the Sun Yat-sen University Cancer Center. Written informed consent was obtained from all individual participants included in the study.

\section{Patient selection}

Patients with pathologically diagnosed and previously untreated rectal cancer in our hospital from January 1st 2007 to March 31st 2014 were initially considered. Those who had pretreatment clinical stage II-III (T3-4N0M0, T1-4N1-2M0) diseases and completed NACRT followed by surgery would be consecutively included for retrospective review of the clinical data.

The exclusion criteria included: (i) age older than 75 years old; (ii) Karnofsky performance score $\leq 70$; (iii) severe heart, lung, liver or kidney dysfunctions unsuitable for NACRT; (iv) prior history of other malignancies; (v) prior chemotherapy or radiotherapy; (vi) DMs during radiotherapy; (vii) application of monoclonal antibody.

\section{Diagnosis and staging work-up}

Before treatment, the patients were all pathologically diagnosed through a rectoscope. And they all underwent a computed tomography (CT) of chest and abdomen, a magnetic resonance imaging (MRI) of pelvis, an endoscopic ultrasonography and a whole-body bone scan or positron emission tomography to evaluate their pretreatment clinical stage. For convenience of analysis, all the patients enrolled were restaged according to the 7th edition of the Union for International Cancer Control / American Joint Cancer Committee (UICC / AJCC) TNM staging classification [13]. The pretreatment carcinoembryonic antigen (CEA) and carbohydrate antigen 19-9 (CA19-9) were also tested for each patient.

\section{Treatment strategies}

The technique of radiotherapy in this study was 3-dimensional conformal radiotherapy (3DCRT), or intensity-modulated radiotherapy (IMRT). As previously described [14], all patients were immobilized at a prone position, using an AIO bellyboard and pelvic solution system (AIO Solution; Orfit Industries, Wijnegem, Belgium). After a simulation based on $\mathrm{CT}$, the target volumes were contoured according to the guidelines of the International Commission on Radiation Units and Measurements Reports 50 and 62. The gross tumor volume (GTV) contained the macroscopic tumor and the enlarged lymph nodes visible on CT or MRI. The clinical target volume (CTV) covered the GTV with a radial margin of $2 \mathrm{~cm}$, and included high-risk regions of lymphatic drainage. If the tumor invaded adjacent structures, a further $1.5-\mathrm{cm}$ isotropic margin into the involved structures would be included in the CTV to cover the microscopic disease. Planning target volumes (PTVs) for GTV and CTV covered the GTV and the CTV with an isotropic margin of $0.6 \mathrm{~cm}$, respectively. A total dose of 5000 cGy and 4600 cGy were given to PTVs for GTV and CTV, respectively. Irradiation was performed in a conventional fractionation (200 cGy per fraction, 1 fraction per day, 5 days per week) and through a linear accelerator delivering an 8-MV photon beam.

The regimen of perioperative chemotherapy was the CAPEOX regimen. Capecitabine was given 1000 $\mathrm{mg} / \mathrm{m}^{2}$ twice daily on Day $1-14$. Oxaliplatin was given $130 \mathrm{mg} / \mathrm{m}^{2}$ on Day $1\left(100 \mathrm{mg} / \mathrm{m}^{2}\right.$ concurrently with radiotherapy). The neoadjuvant chemotherapy 
(NACT) was performed every 21 days, for a total of 2-4 cycles before surgery. The necessity and the cycle number of ACT depended on the decision of the multidisciplinary team consultation. The CAPEOX regimen was taken place by the single-agent capecitabine regimen if: (i) the cumulative dose of oxaliplatin reached $780 \mathrm{mg} / \mathrm{m}^{2}$; (ii) grade $3 / 4$ neural toxicities of the Common Terminology Criteria for Adverse Events (CTCAE) appeared; (iii) CTCAE grade 4 thrombocytopenia appeared twice.

The surgery was scheduled 6-8 weeks after the last cycle of NACT. A radical resection according to the total mesorectal excision standard was performed. When tumor infiltrated or adhered to the adjacent organs, the surgeons would also apply a multivisceral resection, in which partial or total of the attached organs was removed. The postoperative pathology of each patient was re-assessed to decide the pathologic stage, also on basis of the 7th edition of the UICC / AJCC TNM staging classification.

\section{Evaluation of adverse events}

The acute toxicities of NACRT and ACT were evaluated, according to the CTCAE version 4.03. Postoperative complications were also evaluated, based on the Clavien-Dindo classification.

\section{Follow-up}

After treatment, the patients were planned to receive follow-up by outpatient interview every 3-6 months in the first 3 years. The main contents of the outpatient interview included complete physical examination, thoraco-abdominal CT, pelvic MRI, serum CEA and CA19-9 assessment, and annual rectoscope and whole-body bone scan. After the 3rd year, the patients were followed up every 6-12 months by outpatient interview or telephone, until death from rectal cancer, or August 31st 2017, whichever came first. Causes of deaths were confirmed by death certificates.

The primary endpoint of this study was the OS, which was defined as the percentage of patients of a data set who survived after a defined period of time from pathologic diagnosis. The secondary endpoints included the disease-free survival (DFS), the local-recurrence-free survival (RFS) and the distant-metastasis-free survival (MFS). These 3 endpoints were defined as the percentage of patients who had no corresponding events after a certain time period from diagnosis. The events for the DFS included death, LR and DM. And the events for the RFS and the MFS were LR and DM, respectively.

\section{Statistical Analysis}

According to the postoperative pathologic stage, the patients were then divided into 2 groups: the yp0-I group and the ypII-III group. Propensity score matching (PSM) through logistic regression were then performed in these 2 groups, respectively. The matching ratio was 1:1, with chemotherapy cycle $(\leq 7$ vs. $\geq 8)$ as the dependent variable. And the covariates included age, gender (male vs. female), anemia (yes vs. no), tumor differentiation (poorly differentiated vs. moderately-well differentiated), pretreatment clinical stage (cIII vs. cII), CEA, CA19-9, radiation technique (3DCRT vs. IMRT). The cutoff value of the age was the median age of the patients eligible for analysis. The anemia is defined as hemoglobin $<130 \mathrm{~g} / \mathrm{L}$ for male and $<120 \mathrm{~g} / \mathrm{L}$ for female, according to the standard of the World Health Organization [15]. The upper normal limit of serum CEA and CA19-9 were determined as $5.0 \mathrm{ng} / \mathrm{ml}$ and $35 \mathrm{U} / \mathrm{ml}$ respectively, according to the standard of our hospital [16].

In each group, balance of baseline clinicopathological profiles between the patients treated with different cycles of chemotherapy was checked by a Chi-square test, both before and after PSM. The treatment-related complications were compared in the matched patients, also by a Chi-square test.

A Kaplan-Meier approach was then performed to calculate the survivals of the patients treated with different cycles of chemotherapy in the 2 groups, respectively. The patients without death, LR or DM until August 31st 2017, and those lost to follow-up were regarded censored. Difference of the survivals was assessed by a log-rank test.

The whole procedure of statistical analysis was done by IBM SPSS Statistics 23.0 (IBM Co., Armonk, New York, US). A difference with a two-sided $P$ value of less than 0.05 was considered to be statistically significant.

\section{Results}

\section{Patient enrollment}

A total of 1692 consecutive patients were diagnosed with untreated non-metastatic rectal cancer in our hospital, between January 2007 and March 2014. Of those, 1127 patients had stage II-III disease before treatment. Among these LARC patients, 479 cases received NACRT. When the cases with age older than 75 years old $(\mathrm{N}=9)$, DM during treatment $(\mathrm{N}=$ 13), prior malignancies $(\mathrm{N}=23)$, severe comorbidities $(\mathrm{N}=4)$, incomplete NACRT $(\mathrm{N}=20)$ and monoclonal antibody therapy $(\mathrm{N}=22)$ were excluded, there were finally 388 patients eligible for analysis. The procedure of enrollment referred to Figure 1.

The age of the patients ranged from 15 to 75 (median, 55) years old. Thus, the cutoff value of age was 55 years old. The total cycle number of 
perioperative chemotherapy was from 2 to 10 (median, 8). In the patients treated with chemotherapy cycle $\leq 7$, the total cycle number ranged 2-6 (median, 4). And in those treated with chemotherapy cycle $\geq 8$, the total cycle number ranged 8-10 (median, 8) cycles.

\section{Clinicopathological profiles}

The baseline clinicopathological profiles of the patients were showed in Table 1. Before PSM, the numbers of the patients in the yp0-I group were 185 . Compared with those treated with chemotherapy cycle $\leq 7$, there were more cases with stage cIII disease in those treated with chemotherapy cycle $\geq 8(81.1 \%$ vs. $68.4 \%, P=0.045)$. And the numbers of the patients in the ypII-III group were 203. Less cases with age $\geq 55$ years old were seen in those treated with chemotherapy cycle $\geq 8$ (38.1\% vs. $61.1 \%, P=0.001)$, than in those treated with chemotherapy cycle $\leq 7$. After PSM, the numbers of the patients in the yp0-I and the ypII-III groups were 158 and 180, respectively. The baseline clinicopathological characteristics were balanced between the patients treated with different chemotherapy cycles, in either of the 2 groups.

\section{Adverse events}

After PSM, the patients treated with chemotherapy cycle $\geq 8$ appeared to have more acute toxicities than those treated with chemotherapy cycle $\leq 7(83.5 \%$ vs. $57.0 \%, P<0.001)$, in the yp0-I group (Table 1). But no difference was seen in incidence of grade $3 / 4$ acute toxicities or grade 3 postoperative complications between the patients treated with different cycles of chemotherapy.

In the ypII-III group, there was no difference in incidence of acute toxicities, grade $3 / 4$ acute toxicities or grade 3 postoperative complications, between the patients treated with different cycles of chemotherapy (Table 1).

Table 1. Clinicopathological characteristics of the patients.

\begin{tabular}{|c|c|c|c|c|c|c|c|c|c|c|c|c|}
\hline \multirow[b]{4}{*}{ Characteristics } & \multicolumn{6}{|c|}{ Patients with yp0-I disease } & \multicolumn{6}{|c|}{ Patients with ypII-III disease } \\
\hline & \multicolumn{3}{|c|}{ Before PSM (N = 185) } & \multicolumn{3}{|c|}{ After PSM (N = 158) } & \multicolumn{3}{|c|}{ Before PSM (N = 203) } & \multicolumn{3}{|c|}{ After PSM (N = 180) } \\
\hline & \multicolumn{2}{|c|}{ Chemotherapy cycle } & \multirow[b]{2}{*}{$P$ value } & \multicolumn{2}{|c|}{ Chemotherapy cycle } & \multirow[b]{2}{*}{$P$ value } & \multicolumn{2}{|c|}{ Chemotherapy cycle } & \multirow[b]{2}{*}{$P$ value } & \multicolumn{2}{|c|}{ Chemotherapy cycle } & \multirow[b]{2}{*}{$P$ value } \\
\hline & $\leq 7$ & $\geq 8$ & & $\leq 7$ & $\geq 8$ & & $\leq 7$ & $\geq 8$ & & $\leq 7$ & $\geq 8$ & \\
\hline \multicolumn{13}{|l|}{ Age/years old } \\
\hline$\geq 55$ & $43(54.4 \%)$ & $53(50.0 \%)$ & 0.551 & $43(54.4 \%)$ & $36(45.6 \%)$ & 0.265 & $55(61.1 \%)$ & $43(38.1 \%)$ & $0.001^{* *}$ & $55(61.1 \%)$ & $43(47.8 \%)$ & 0.073 \\
\hline$<55$ & $36(45.6 \%)$ & $53(50.0 \%)$ & & $36(45.6 \%)$ & $43(54.4 \%)$ & & $35(38.9 \%)$ & $70(61.9 \%)$ & & $35(38.9 \%)$ & $47(52.2 \%)$ & \\
\hline \multicolumn{13}{|l|}{ Gender } \\
\hline Male & $50(63.3 \%)$ & $67(63.2 \%)$ & 0.991 & $50(63.3 \%)$ & $49(62.0 \%)$ & 0.869 & $67(74.4 \%)$ & $74(65.5 \%)$ & 0.169 & $67(74.4 \%)$ & $63(70.0 \%)$ & 0.506 \\
\hline Female & $29(36.7 \%)$ & $39(36.8 \%)$ & & $29(36.7 \%)$ & $30(38.0 \%)$ & & $23(25.6 \%)$ & $39(34.5 \%)$ & & $23(25.6 \%)$ & $27(30.0 \%)$ & \\
\hline \multicolumn{13}{|l|}{ Anemia } \\
\hline Yes & $19(24.1 \%)$ & $27(25.5 \%)$ & 0.825 & $19(24.1 \%)$ & $22(27.8 \%)$ & 0.586 & $34(37.8 \%)$ & $41(36.3 \%)$ & 0.827 & $34(37.8 \%)$ & $35(38.9 \%)$ & 0.878 \\
\hline No & $60(75.9 \%)$ & $79(74.5 \%)$ & & $60(75.9 \%)$ & $57(72.2 \%)$ & & $56(62.2 \%)$ & $72(63.7 \%)$ & & $56(62.2 \%)$ & $55(61.1 \%)$ & \\
\hline \multicolumn{13}{|l|}{ Differentiation } \\
\hline Poorly & $9(11.4 \%)$ & $11(10.4 \%)$ & 0.826 & $9(11.4 \%)$ & $9(11.4 \%)$ & 1.000 & $14(15.6 \%)$ & $17(15.0 \%)$ & 0.920 & $14(15.6 \%)$ & $11(12.2 \%)$ & 0.518 \\
\hline $\begin{array}{l}\text { Moderately- } \\
\text { well }\end{array}$ & $70(88.6 \%)$ & $95(89.6 \%)$ & & $70(88.6 \%)$ & $70(88.6 \%)$ & & $76(84.4 \%)$ & $96(85.0 \%)$ & & $76(84.4 \%)$ & $79(87.8 \%)$ & \\
\hline \multicolumn{13}{|l|}{$\mathrm{CEA} / \mathrm{ng} / \mathrm{ml}$} \\
\hline$\geq 5$ & $24(30.4 \%)$ & $39(36.8 \%)$ & 0.363 & $24(30.4 \%)$ & $27(34.2 \%)$ & 0.610 & $51(56.7 \%)$ & $59(52.2 \%)$ & 0.527 & $51(56.7 \%)$ & $51(56.7 \%)$ & 1.000 \\
\hline$<5$ & $55(69.6 \%)$ & $67(61.2 \%)$ & & $55(69.6 \%)$ & $52(63.8 \%)$ & & $39(43.3 \%)$ & $54(47.8 \%)$ & & $39(43.3 \%)$ & $39(43.3 \%)$ & \\
\hline \multicolumn{13}{|l|}{ CA19-9/U/ml } \\
\hline$\geq 35$ & $9(11.4 \%)$ & $15(14.2 \%)$ & 0.581 & $9(11.4 \%)$ & $10(12.7 \%)$ & 0.807 & $24(26.7 \%)$ & $19(16.8 \%)$ & 0.088 & $24(26.7 \%)$ & $19(21.1 \%)$ & 0.382 \\
\hline$<35$ & $70(88.6 \%)$ & $91(85.8 \%)$ & & $70(88.6 \%)$ & $69(87.3 \%)$ & & $66(73.3 \%)$ & $94(83.2 \%)$ & & $66(73.3 \%)$ & $71(78.9 \%)$ & \\
\hline \multicolumn{13}{|l|}{ Clinical stage } \\
\hline cIII & $54(68.4 \%)$ & $86(81.1 \%)$ & $0.045^{*}$ & $54(68.4 \%)$ & $61(77.2 \%)$ & 0.211 & $76(84.4 \%)$ & $97(85.8 \%)$ & 0.781 & $76(84.4 \%)$ & $76(84.4 \%)$ & 1.000 \\
\hline cII & $25(31.6 \%)$ & $20(18.9 \%)$ & & $25(31.6 \%)$ & $18(22.8 \%)$ & & $14(15.6 \%)$ & $16(14.2 \%)$ & & $14(15.6 \%)$ & $14(15.6 \%)$ & \\
\hline \multicolumn{13}{|c|}{ Radiation technique } \\
\hline 3DCRT & $27(34.2 \%)$ & $43(40.6 \%)$ & 0.375 & $27(34.2 \%)$ & $36(45.6 \%)$ & 0.144 & $42(46.7 \%)$ & $51(45.1 \%)$ & 0.828 & $42(46.7 \%)$ & $50(55.6 \%)$ & 0.233 \\
\hline IMRT & $52(65.8 \%)$ & $63(59.4 \%)$ & & $52(65.8 \%)$ & $43(54.4 \%)$ & & $48(53.3 \%)$ & $62(54.9 \%)$ & & $48(53.3 \%)$ & $40(44.4 \%)$ & \\
\hline \multicolumn{13}{|l|}{ Acute toxicities } \\
\hline Yes & & & & $45(57.0 \%)$ & $66(83.5 \%)$ & $<0.001^{* *}$ & & & & $31(34.4 \%)$ & $38(42.2 \%)$ & 0.283 \\
\hline No & & & & $34(43.0 \%)$ & $13(16.5 \%)$ & & & & & $59(65.6 \%)$ & $52(57.8 \%)$ & \\
\hline Grade $3 / 4$ acute & toxicities & & & & & & & & & & & \\
\hline Yes & & & & $9(11.4 \%)$ & $16(20.3 \%)$ & 0.127 & & & & $10(11.1 \%)$ & $11(12.2 \%)$ & 0.816 \\
\hline No & & & & $70(88.6 \%)$ & $63(79.7 \%)$ & & & & & $80(88.9 \%)$ & $79(87.8 \%)$ & \\
\hline Postsurgical cor & mplications & & & & & & & & & & & \\
\hline Grade 3 & & & & $3(3.8 \%)$ & $2(2.5 \%)$ & 0.649 & & & & $3(3.3 \%)$ & $4(4.4 \%)$ & 0.700 \\
\hline Grade $0-2$ & & & & $76(96.2 \%)$ & 77 (97.5\%) & & & & & 87 (96.7\%) & $86(95.6 \%)$ & \\
\hline
\end{tabular}




\section{Survival analysis}

Totally 20 out of the 388 patients (5.2\%) were lost to follow-up. The median follow-up time was 55 (range, 10-120) months. In the whole cohort of the matched patients, chemotherapy cycle $\geq 8$ appeared to be associated with a better OS $(81.7 \%$ vs. $68.6 \%, P=$ $0.003)$, DFS (75.1\% vs. $59.8 \%, P=0.002)$ and MFS (75.7\% vs. $62.1 \%, P=0.004)$.

In the ypII-III group, the patients treated with chemotherapy cycle $\geq 8$ appeared to have a better OS (75.6\% vs. $57.8 \%, P=0.006)$, DFS $(68.9 \%$ vs. $48.9 \%, P=$ $0.005)$ and MFS (68.9\% vs. $52.2 \%, P=0.014)$, compared with those treated with chemotherapy cycle $\leq 7$. No difference was seen in the RFS between the patients treated with different cycles of chemotherapy.

Oppositely, in the yp0-I group, no significant difference was seen in the OS $(88.6 \%$ vs. $81.0 \%, P=$ $0.150)$, the DFS $(94.9 \%$ vs. $94.9 \%, P=0.996)$, the RFS $(83.5 \%$ vs. $73.4 \%, P=0.122)$ or the MFS $(82.3 \%$ vs. $72.2 \%, P=0.150)$, between the patients treated with different cycles of chemotherapy.

The survival curves of the whole cohort, the

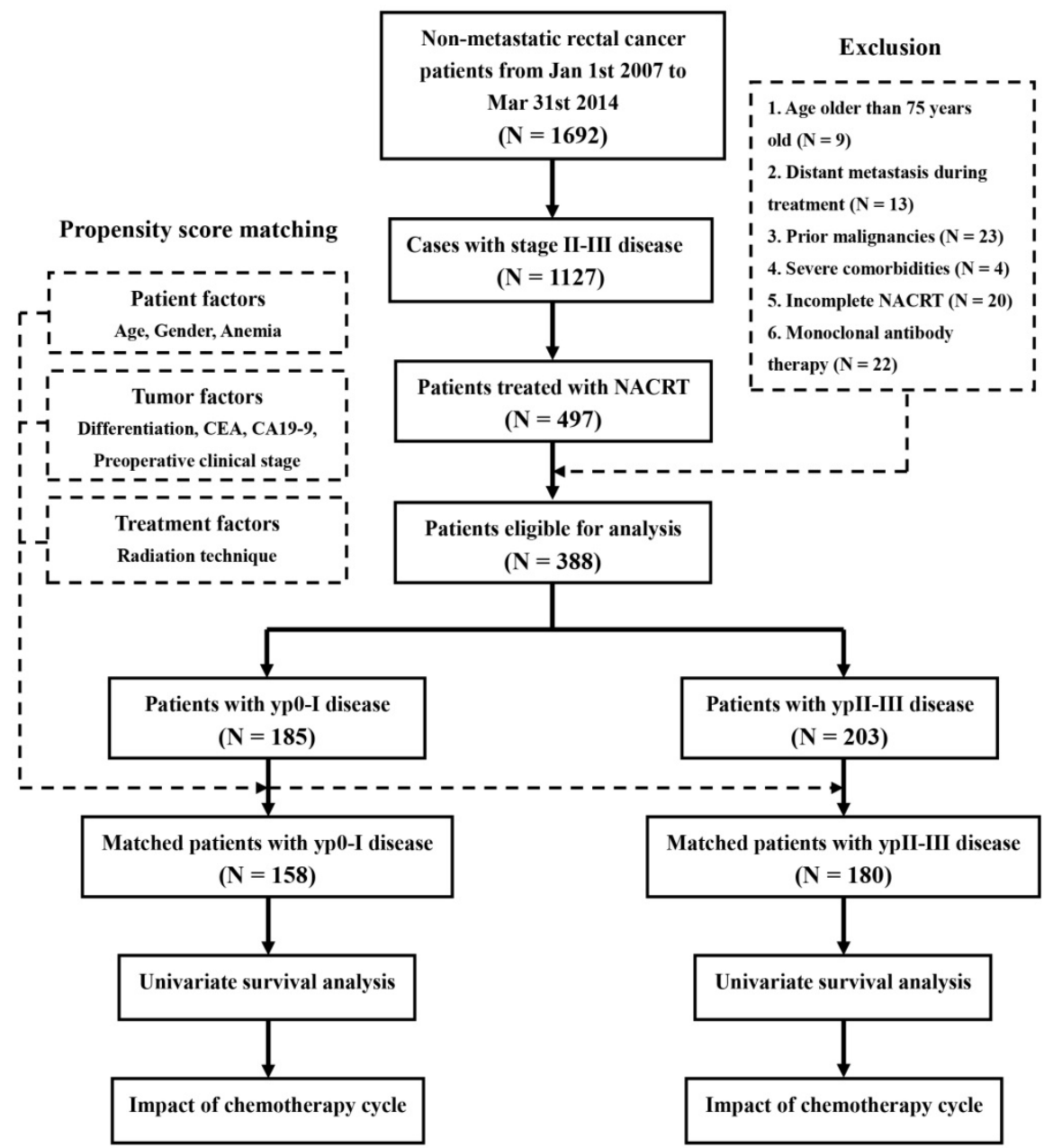

Figure 1. Procedure of enrollment and analysis. Abbrevations: NACRT, neoadjuvant chemoradiotherapy; CEA, carcinoembryonic antigen; CA19-9, carbohydrate antigen 19-9.
ypII-III and the yp0-I groups after PSM were shown in Figures 2-4, respectively.

\section{Discussion}

Many experiences of chemotherapy regimens and intensity for rectal cancer come from colon cancer $[11,17]$, because of the similarity on pathologic types $[18,19]$. Nevertheless, even for colon cancer, there are few studies focusing on relationship between the cycle number of perioperative chemotherapy and the prognosis of patients [20,21]. And based on these studies, the impact of chemotherapy cycle on clinical outcomes is uncertain. Moreover, there is no direct evidence on the appropriate chemotherapy cycle for LARC patients until now. After balancing the familiar prognosticators through PSM, a widely accepted approach to control selection bias in observational studies [22], we demonstrated that chemotherapy cycle was associated with the prognosis of LARC patients, especially those with late (ypII-III) postoperative pathologic stage, even under the current standard mode of treatment. CAPEOX chemotherapy of at least 8 cycles seemed to be effective enough to improve the OS of both the whole cohort of patients (from $68.6 \%$ to $81.7 \%, P=0.003)$, and the patients with ypII-III disease (from $57.8 \%$ to $75.6 \%, P=0.006$ ). Considering the CAPEOX has been gradually accepted as the standard regimen of chemotherapy for LARC, this finding may be informative for clinicians to perform treatment strategies and conduct clinical trials.

The LARC has a propensity of DM. In this study, the DM rates of the whole cohort, the yp0-I and the ypII-III groups were $31.1 \%$, $21.5 \%$ and $39.4 \%$ respectively, after a median follow-up of 55 months. Yet, the LR rates were only $6.5 \%$, $5.1 \%$ and $7.8 \%$, respectively. Hence, it was more suitable to consider LARC as a systemic disease, for which intensified systemic chemotherapy was needed. It has already been proved that prolonged cycles of chemotherapy could improve the survival of patients in many solid tumors which are prone to metastasize, such as breast, ovarian and nasopharyngeal 
cancers [23-25]. Tsai et al also reported in colon cancer that at least 8 cycles of ACT with the mFOLFOX6 regimen was needed to reduce $\mathrm{DM}$ and improve prognosis. Although no difference of RFS was seen in our study between the LARC patients treated with different cycles of chemotherapy, the difference of MFS was obvious $(75.7 \%$ vs. $62.1 \%, P=0.004)$, especially in the cases with ypII-III disease $(68.9 \%$ vs. $52.2 \%, P=0.014)$. In other words, reduction of DM through perioperative chemotherapy of $\geq 8$ cycles might be the reason of survival improvement in these patients. On the contrary, a recent phase 3 trial of the
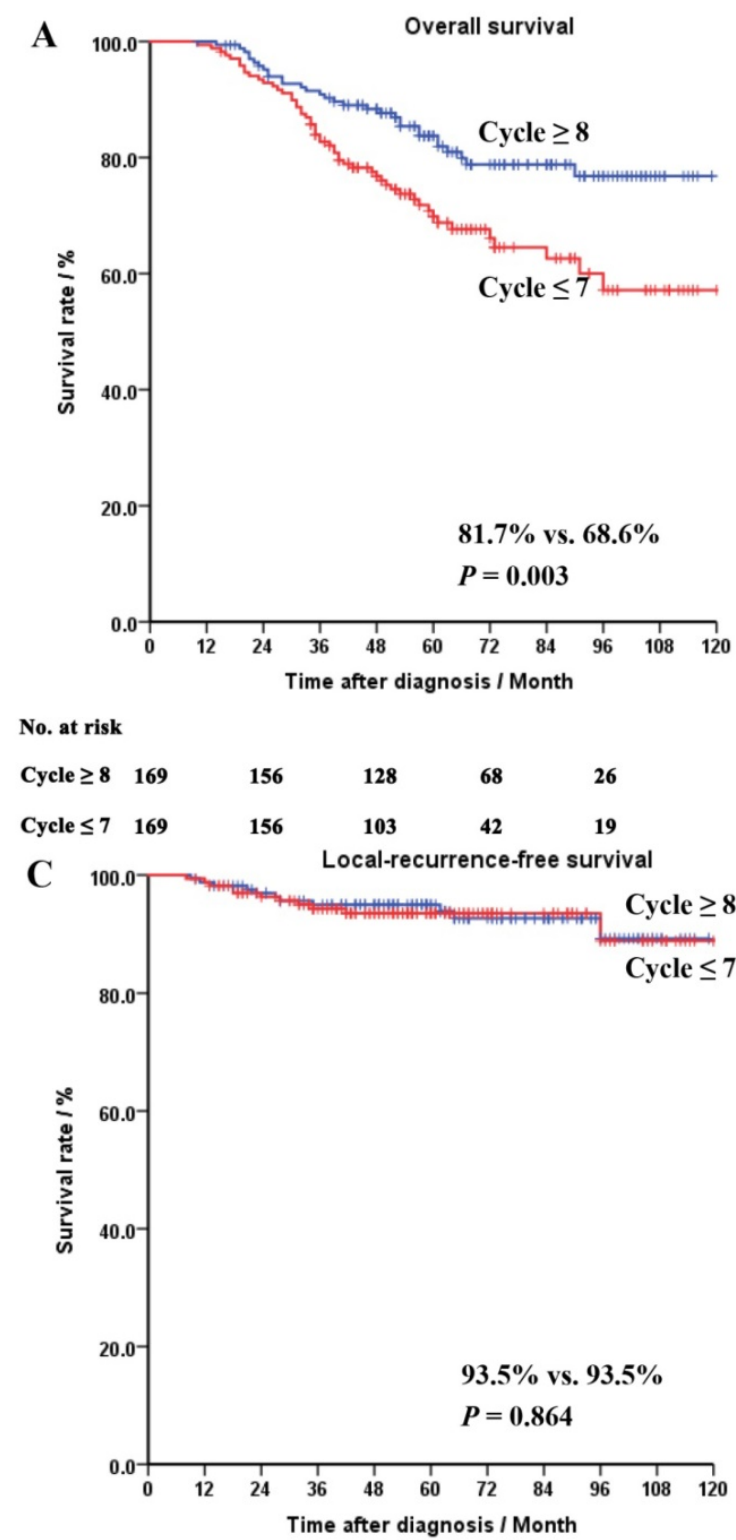

No. at risk

$\begin{array}{llllll}\text { Cycle } \geq 8 & 169 & 153 & 123 & 66 & 24 \\ \text { Cycle } \leq 7 & 169 & 150 & 102 & 41 & 18\end{array}$
Dutch Colorectal Cancer Group showed that when combined with NACRT and surgery, ACT of 8 cycles was not statistically superior to observation in managing LARC [26]. However, a nearly 7\% difference of DFS was seen between the 2 groups of patients. Because the trial was closed prematurely, the authors considered that the difference would be significant when statistical power was appropriate. It could still be inferred that chemotherapy of 8 cycles or more might have a potential to improve the prognosis of LARC.
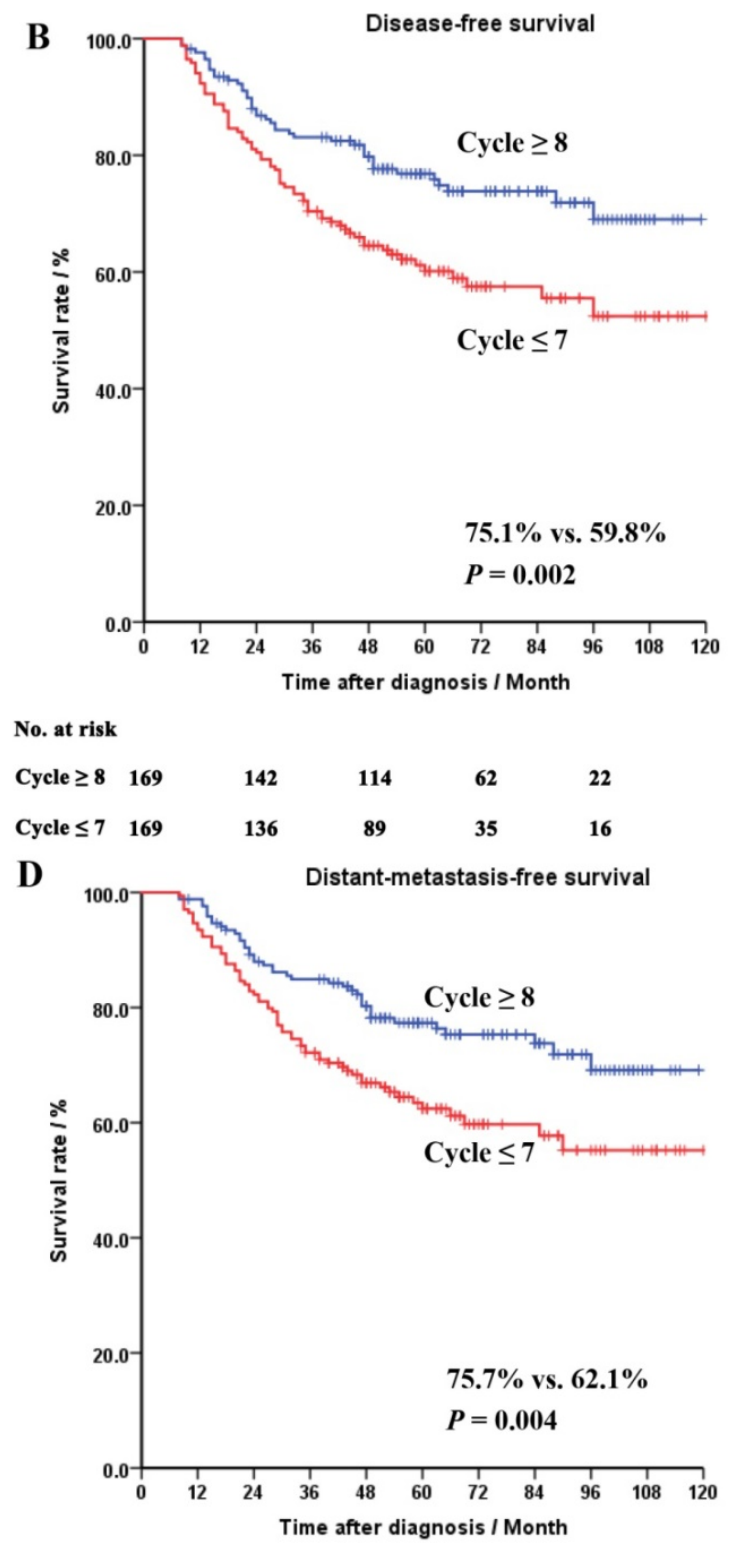

No. at risk

$\begin{array}{lllccr}\text { Cycle } \geq 8 & 169 & 144 & 115 & 64 & 23 \\ \text { Cycle } \leq 7 & 169 & 139 & 90 & 36 & 17\end{array}$

Figure 2. Survival curves of the whole cohort of the matched patients. Panel A: overall survival. Panel B: disease-free survival. Panel C: local-recurrence-free survival. Panel D: distant-metastasis-free survival. 

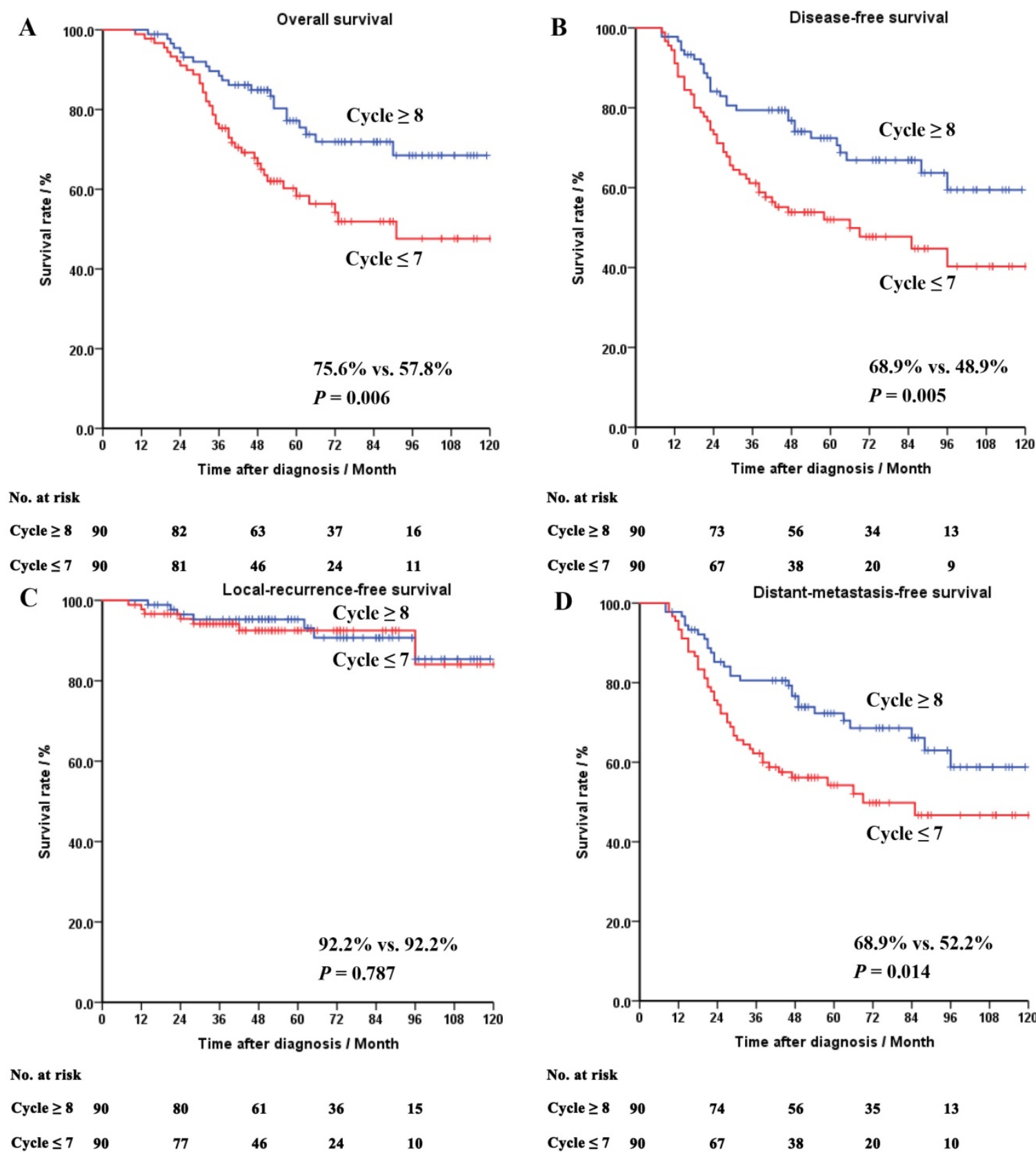

Figure 3. Survival curves of the matched patients in the ypll-III group. Panel A: overall survival. Panel B: disease-free survival. Panel C: local-recurrence-free survival. Panel D: distant-metastasis-free survival.

Admittedly, the influence of chemotherapy on life quality is another concern. According to a Phase 3 trial by Schmoll et al, acute toxicities were seen in $98 \%$ of the patients who received 8 cycles of CAPEOX. And among these acute toxicities, $56.5 \%$ were of grade $3 / 4$ [11]. Our study also showed that 104 out of the 169 matched patients (61.5\%) receiving CAPEOX of $\geq$ 8 cycles presented with a chemotherapy-related toxicity. So it is necessary to screen out the patients really at high risk of DM and in need of intensified chemotherapy. As we know, the treatment effect of NACRT is one of the most important factors determining the prognosis of LARC $[7,27]$. It is easily inferred that the patients who had greater residual tumor after NACRT might benefit more from chemotherapy of prolonged cycles. Oppositely, prolonged chemotherapy might bring less benefit, or even cause harm, to those who had smaller or no residual tumor. In our study, chemotherapy of 8 cycles or more did not improve the survivals of the patients with yp0-I disease, but only increase the incidence of acute toxicities $(83.5 \%$ vs. $57.0 \%, P<$ $0.001)$. These results confirmed our hypothesis. Therefore, we suggested that patients with early (yp0-I) postoperative pathologic stage were at low risk of DM and might not be the suitable population 
for high-intensity chemotherapy. Similar to our study, population-based studies by Maas et al and $\mathrm{Hu}$ et al revealed that patients exhibiting a complete pathologic response after NACRT attained no benefit from ACT [28,29]. Interestingly, a study by Collette et al achieved a different result that patients who had ypT0-2 disease and were considered sensitive to NACRT seemed to benefit from ACT [30].

Indeed, this study had 2 main shortcomings.

First, it was a retrospective study. The nature of the
A

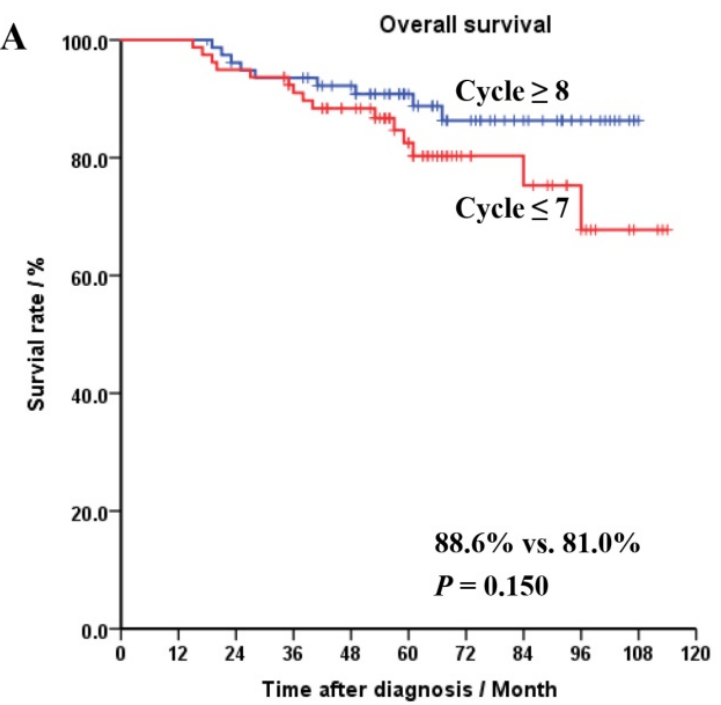

study might bring some biases, such as selection biases. But PSM is convinced to be a method that could balance the confounding factors and control the biases effectively [22]. Second, the sample size was relatively small. It was because the proportion of the LARC patients who received NACRT was not large before the year of 2012, even in the developed countries [3]. A prospective randomized controlled trail enrolling a large sample of patients is needed to validate the results of our study.

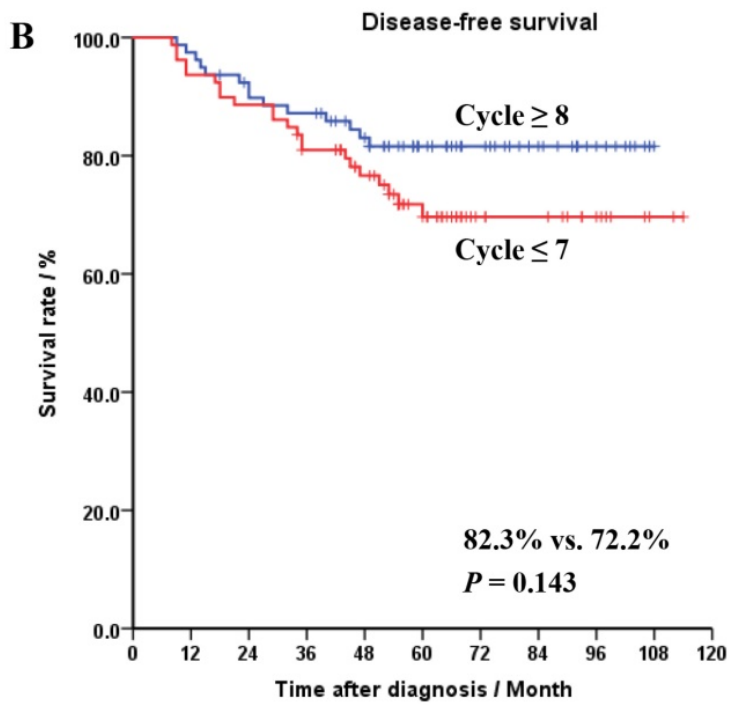

No. at risk

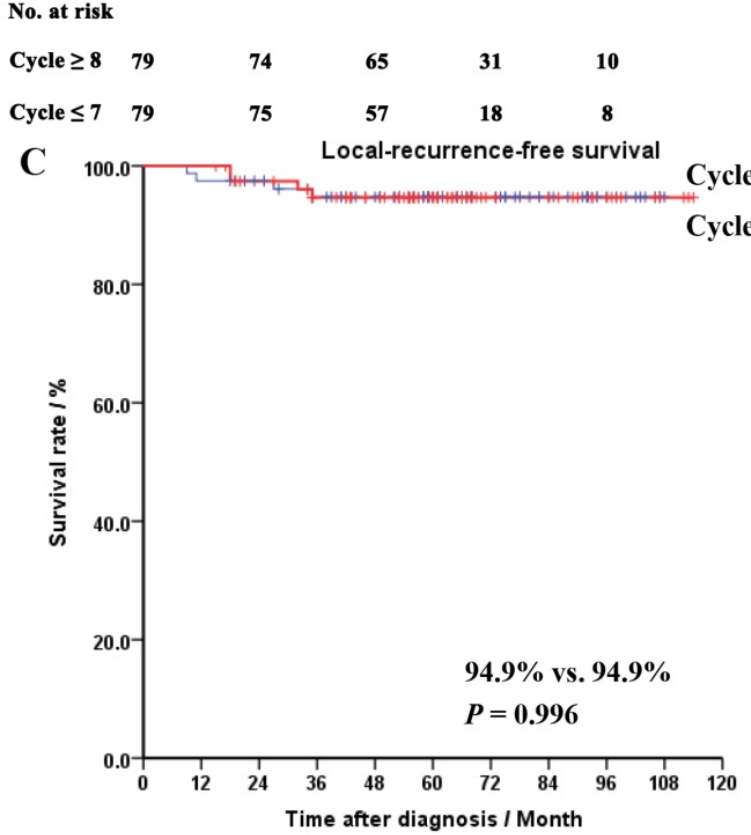

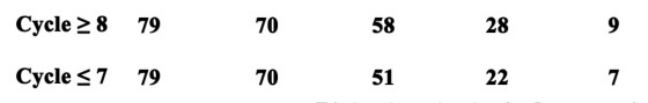

Distant-metastasis-free sur
Cycle $\geq 8$

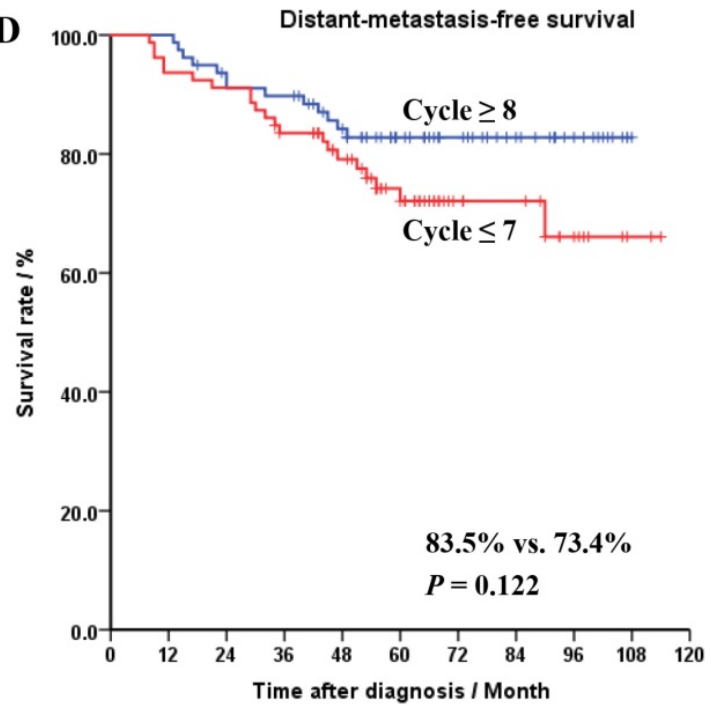

No. at risk

\begin{tabular}{|c|c|c|c|c|}
\hline Cycle $\geq 8$ & 79 & 70 & 59 & 29 \\
\hline Cycle $\leq 7$ & 79 & 72 & 52 & 16 \\
\hline
\end{tabular}

No. at risk

\begin{tabular}{|c|c|c|c|c|}
\hline Cycle $\geq 8$ & 79 & 73 & 62 & 30 \\
\hline Cycle $\leq 7$ & 79 & 73 & 56 & 17 \\
\hline
\end{tabular}

Figure 4. Survival curves of the matched patients in the yp0-I group. Panel A: overall survival. Panel B: disease-free survival. Panel C: local-recurrence-free survival. Panel D: distant-metastasis-free survival. 


\section{Conclusion}

Perioperative chemotherapy of 8 cycles or more appeared to improve the survival of LARC patients, especially those with ypII-III disease. But prolonged chemotherapy should be performed with caution in patients with yp0-I stage. This finding is informative though further verification is needed.

\section{Abbreviations}

NACT, neoadjuvant chemoradiotherapy; ACT, adjuvant chemotherapy; NCCN, National Comprehensive Cancer Network; OS, overall survival; LARC, locoregionally advanced rectal cancer; DM, distant metastasis; LR, local recurrence; CT, computed tomography; MRI, magnetic resonance imaging; UICC/AJCC, International Cancer Control/American Joint Cancer Committee; CEA, carcinoembryonic antigen; CA19-9, carbohydrate antigen 19-9; 3DCRT, 3-dimensional conformal radiotherapy; IMRT, intensity-modulated radiotherapy; GTV, gross tumor volume; CTV, clinical target volume; PTV, planning target volume; NACT, neoadjuvant chemotherapy; CTCAE, Common Terminology Criteria for Adverse Events; DFS, disease-free survival; RFS, localrecurrence-free survival; MFS, distant-metastasis-free survival; PSM, propensity score matching.

\section{Competing Interests}

The authors have declared that no competing interest exists.

\section{References}

1. Chen $\mathrm{W}$, Sun $\mathrm{K}$, Zheng R, et al. Cancer incidence and mortality in China, 2014. Chin J Cancer Res. 2018; 30(1): 1-12.

2. Liu $\mathrm{F}$, $\mathrm{Li} \mathrm{C}$, Jia $\mathrm{H}$, et al. Is there a prognostic value of tumor location among Chinese patients with colorectal cancer? Oncotarget. 2017; 8(24): 38682-38692.

3. Sineshaw HM, Jemal A, Thomas CR Jr, et al. Changes in treatment patterns for patients with locally advanced rectal cancer in the United States over the past decade: An analysis from the National Cancer Data Base. Cancer. 2016; 122(13): 1996-2003.

4. Deng Y, Chi P, Lan P, et al. Modified FOLFOX6 With or Without Radiation Versus Fluorouracil and Leucovorin With Radiation in Neoadjuvant Treatment of Locally Advanced Rectal Cancer: Initial Results of the Chinese FOWARC Multicenter, Open-Label, Randomized Three-Arm Phase III Trial. J Clin Oncol. 2016; 34(27): 3300-3307.

5. Cassidy RJ, Liu Y, Patel $\mathrm{K}$, et al. Can we eliminate neoadjuvant chemoradiotherapy in favor of neoadjuvant multiagent chemotherapy for select stage II/III rectal adenocarcinomas: Analysis of the National Cancer Database. Cancer. 2017; 123(5): 783-793.

6. [Internet] NCCN.org. NCCN Clinical Practice Guidelines in Oncology (NCCN Guidelines $\left.{ }^{\circledR}\right)$ : Rectal Cancer. (Version 3. 2017). Available at: https://www.nccn.org/store/login/login.aspx?ReturnURL=https://www. nccn.org/professionals/physician_gls/pdf/rectal.pdf. (Accessed March 13, 2017).

7. Fokas E, Liersch T, Fietkau R, et al. Tumor regression grading after preoperative chemoradiotherapy for locally advanced rectal carcinoma revisited: updated results of the CAO/ARO/AIO-94 trial. J Clin Oncol. 2014; 32(15): 1554-1562.

8. Sclafani F, Brown G, Cunningham D, et al. PAN-EX: a pooled analysis of two trials of neoadjuvant chemotherapy followed by chemoradiotherapy in MRI-defined, locally advanced rectal cancer. Ann Oncol. 2016; 27(8): 1557-1565.

9. Rödel C, Graeven U, Fietkau R, et al. Oxaliplatin added to fluorouracil-based preoperative chemoradiotherapy and postoperative chemotherapy of locally advanced rectal cancer (the German CAO/ARO/AIO-04 study): final results of the multicentre, open-label, randomised, phase 3 trial. Lancet Oncol. 2015; 16(8): 979-989.
10. Gupta GP, Massagué J. Cancer metastasis: building a framework. Cell. 2006; 127(4): 679-695.

11. Schmoll HJ, Cartwright T, Tabernero J, et al. Phase III trial of capecitabine plus oxaliplatin as adjuvant therapy for stage III colon cancer: a planned safety analysis in 1,864 patients. J Clin Oncol. 2007; 25(1): 102-109.

12. Magni E, Botteri E, Ravenda PS, et al. Detection of circulating tumor cells in patients with locally advanced rectal cancer undergoing neoadjuvant therapy followed by curative surgery. Int J Colorectal Dis. 2014; 29(9): 1053-1059.

13. Sobin LH, Gospodarowicz MK, Wittekind C. TNM Classification of Malignant Tumours (UICC International Union Against Cancer), 7th edition. New York, USA: Wiley-Blackwell; 2009.

14. Gao $\mathrm{YH}, \mathrm{Lin} \mathrm{JZ}$, An $\mathrm{X}$, et al. Neoadjuvant sandwich treatment with oxaliplatin and capecitabine administered prior to, concurrently with, and following radiation therapy in locally advanced rectal cancer: a prospective phase 2 trial. Int J Radiat Oncol Biol Phys. 2014; 90(5): 1153-1160.

15. World Health Organization. The Global Prevalence of Anaemia In 2011. Geneva Switzerland WHO. 2011; 126(11): 5409-5418.

16. Lu Z, Peng J, Wang Z, et al. High preoperative serum CA19-9 level is predictive of poor prognosis for patients with colorectal liver oligometastases undergoing hepatic resection. Med Oncol. 2016; 33(11): 121.

17. Haller DG, Tabernero J, Maroun J, et al. Capecitabine plus oxaliplatin compared with fluorouracil and folinic acid as adjuvant therapy for stage III colon cancer. J Clin Oncol. 2011; 29(11): 1465-1471.

18. Thuraisingam R, Jandova J, Pandit V, et al. Assessing the national trends in colon cancer among Native Americans: A 12 year SEER database study. Am J Surg. 2017; 214(2): 228-231.

19. Huang C, Shen JC, Zhang J, et al. Clinical comparison of laparoscopy vs open surgery in a radical operation for rectal cancer: A retrospective case-control study. World J Gastroenterol. 2015; 21(48):13532-13541.

20. Tsai YJ, Lin JK, Chen WS, et al. Adjuvant FOLFOX treatment for stage III colon cancer: how many cycles are enough? Springerplus. 2016; 5(1): 1318

21. Kumar A, Peixoto RD, Kennecke HF, et al. Effect of Adjuvant FOLFOX Chemotherapy Duration on Outcomes of Patients With Stage III Colon Cancer. Clin Colorectal Cancer. 2015; 14(4): 262-268.

22. Reeder-Hayes KE, Meyer AM, Hinton SP, et al. Comparative Toxicity and Effectiveness of Trastuzumab-Based Chemotherapy Regimens in Older Women With Early-Stage Breast Cancer. J Clin Oncol. 2017; 35(29): 3298-3305.

23. Pinedo HM, de Gruijl TD, van Der Wall E, et al. Biological concepts of prolonged neoadjuvant treatment plus GM-CSF in locally advanced tumors. Oncologist. 2000; 5(6): 497-500.

24. da Costa Miranda V, de Souza Fêde ÂB, Dos Anjos $\mathrm{CH}$, et al. Neoadjuvant chemotherapy with six cycles of carboplatin and paclitaxel in advanced ovarian cancer patients unsuitable for primary surgery: Safety and effectiveness. Gynecol Oncol. 2014; 132(2): 287-291.

25. Guo SS, Tang LQ, Zhang L, et al. The impact of the cumulative dose of cisplatin during concurrent chemoradiotherapy on the clinical outcomes of patients with advanced-stage nasopharyngeal carcinoma in an era of intensity-modulated radiotherapy. BMC Cancer. 2015; 15: 977.

26. Breugom AJ, van Gijn W, Muller EW, et al. Adjuvant chemotherapy for rectal cancer patients treated with preoperative (chemo)radiotherapy and total mesorectal excision: a Dutch Colorectal Cancer Group (DCCG) randomized phase III trial. Ann Oncol. 2015; 26(4): 696-701.

27. Park IJ, You YN, Agarwal A, et al. Neoadjuvant treatment response as an early response indicator for patients with rectal cancer. J Clin Oncol. 2012; 30(15):1770-1776.

28. Maas M, Nelemans PJ, Valentini V, et al. Adjuvant chemotherapy in rectal cancer: defining subgroups who may benefit after neoadjuvant chemoradiation and resection: a pooled analysis of 3,313 patients. Int J Cancer. 2015; 137(1): 212-220.

29. Hu X, Li YQ, Li QG, et al. Adjuvant Chemotherapy Seemed Not to Have Survival Benefit in Rectal Cancer Patients with ypTis-2N0 After Preoperative Radiotherapy and Surgery from a Population-Based Propensity Score Analysis. Oncologist. 2018; [Epub ahead of print].

30. Collette L, Bosset JF, den Dulk M, et al. Patients with curative resection of cT3-4 rectal cancer after preoperative radiotherapy or radiochemotherapy: does anybody benefit from adjuvant fluorouracil-based chemotherapy? A trial of the European Organisation for Research and Treatment of Cancer Radiation Oncology Group. J Clin Oncol. 2007; 25(28): 4379-4386. 